\title{
Reconsideration of urine culture for the diagnosis of acute pyelonephritis in children: a new challenging method for diagnosing acute pyelonephritis
}

Jun Ho Lee, MD, PhD, Seonkyeong Rhie, MD

Department of Pediatrics, CHA Bundang Medical Center, CHA University School of Medicine, Seongnam, Korea

Acute pyelonephritis (APN) should be detected and treated as soon as possible to reduce the risk of the development of acquired renal scarring. However, in the medical field, urine culture results are not available or considered when the prompt discrimination of APN is necessary and empirical treatment is started. Furthermore, urine culture cannot discriminate APN among children with febrile urinary tract infection (UTI) (pyelitis, lower UTI with other fever focus). Therefore, the usefulness of urine culture for diagnostic purposes is small and the sampling procedure is invasive. Congenital hypoplastic kidney is the most common cause of chronic kidney injury in children. Thus, it is desirable that a main target be detected as early as possible when imaging studies are performed in children with APN. However, if APN does not recur, no medical or surgical treatment or imaging studies would be needed because the acquired renal scar would not progress further. Therefore, the long-term prognosis of APN in young children, particularly infants, depends on the number of recurrent APN, not other febrile UTI. New methods that enable prompt, practical, and comfortable APN diagnosis in children are needed as alternatives to urinary catheterization for urine culture sampling.

Key words: Pyelonephritis, Pyelitis, Urine culture, Congenital hypoplastic kidney, Acquired renal scarring

\section{Introduction}

The diagnosis of acute pyelonephritis (APN) depends on urine culture results for confirmation. ${ }^{1)}$ However, urine culture alone is unacceptable as the major determining factor of the diagnosis of APN in children for the following reasons. First, the urine culture results are not available or considered when the prompt discrimination of APN is required in the medical field. Second, about 10\%-35\% of APN cases confirmed with acute dimercaptosuccinic acid (DMSA) scans have revealed negative urine culture results. ${ }^{2,3)}$ Third, urinalysis alone has the highest sensitivity (about 100\%) and negative predictive value (94.7\%) among the methods used for the diagnosis of urinary tract infection (UTI), although it also has a high false positive rate and low positive predictive value. ${ }^{4)}$ Fourth, about $1 \%-3 \%$ of the healthy population has asymptomatic bacteriuria that persists for several months. ${ }^{5}$ Fifth, the empirical treatment of APN should be initiated as soon as possible after its clinical diagnosis to reduce the risk of renal scarring before the urine culture results are revealed. Sixth, procedures like urethral catheterization used to collect appropriate urine culture samples are very invasive for many children who respond well to empirical UTI treatment. And finally, the urine culture results cannot discriminate APN that leads to renal scarring from febrile UTI (fUTI). ${ }^{3)}$
Corresponding author: Seonkyeong Rhie, MD Department of Pediatrics, CHA Bundang Medical Center, CHA University, 59 Yatap-ro, Bundang-gu, Seongnam 13496, Korea

Tel: $+82-31-780-5230$

Fax: +82-31-780-5011

E-mail: starclusters@gmail.com https://orcid.org/0000-0003-3371-8310

Received: 25 June, 2019

Revised: 28 September, 2019

Accepted: 15 October, 2019

See commentary "Is there a simple and less invasive way to accurately diagnose acute pyelonephritis?" in Volume 62 on page 442

Copyright @ 2019 by The Korean Pediatric Society

This is an open-access article distributed under the terms of the Creative Commons Attribution NonCommercial License (http://creativecommons.org/ licenses/by-nc/4.0/n which permits unrestricted noncommercial use, distribution, and reproduction in any medium, provided the original work is properly cited. 
APN should be detected early to reduce the risk of developing acquired renal scarring. ${ }^{6)}$ Acquired renal scarring generally develops in 10\%-30\% of children with first episode of APN, and its incidence increases exponentially as the number of APN recurrences increases. ${ }^{6}$ The renal outcome of children with acquired renal scarring is not clearly known. ${ }^{7)}$ However, if more than half of the normal glomeruli in the renal parenchyma are damaged and cause acquired renal scarring due to recurrent APN, chronic kidney disease (CKD) will develop. Therefore, APN that recurs many times leads to renal scarring and CKD. In young children, particularly those under 24 months of age who have a high incidence rate of APN due to an immunocompromised state as well as a lack of a defense mechanism, are too young to develop CKD caused by recurrent APN. Therefore, it is reasonable that the main targets of UTI imaging studies in young children are the presence of congenital scarring (or hypoplastic kidney) and the recurrence of APN, not the presence of vesicoureteral reflux (VUR).

It is well known that congenital hypoplastic kidney is the most common cause of CKD in children. ${ }^{8)}$ Therefore, the early detection of congenital hypoplastic kidney or congenital renal scarring is considered the most important factor in the management or monitoring of young children with APN.

\section{Urinalysis is the most important tool in the diagnosis of APN}

Abnormal urinalysis and high fever $\left(\geq 38^{\circ} \mathrm{C}\right)$ are the most important factors in the diagnosis of APN. Children with APN showing normal urinalysis findings are rare, particularly young children less than 24 months of age because they have no bladder symptoms that can lead to false negative urinalysis results.

A urinalysis is conducted using a urine dipstick test and a urine microscopic exam. In the urine microscopic exam, pyuria (white blood cells $[\mathrm{WBCs}] \geq 5$ per high-power field) is necessary for the initial diagnosis of APN. Leukocyte esterase (LE) on a urine dipstick test is typically positive in children with pyuria. Only the LE-positive finding on urinalysis is often false positive, meaning it is not due to UTI. The authors named it the "full house" sign of UTI on urinalysis that reveals positive findings on all of the following: occult blood, protein, nitrite, and LE on a dipstick test as well as WBC and red blood cells on a microscopic exam. Alongside pyuria and LE positivity, more than one of those full house signs strongly suggests UTI.

In uncircumcised males less than 24 months of age, only pyuria and LE positivity strongly suggest UTI; in females of the same age or circumcised males, those 2 positive findings are suggestive of aseptic pyuria like vaginitis, balanitis, or a viral infection. This is because both uncircumcised males and those less than 24 months of age have high risk factors of UTI.

However, positive urinalysis findings and positive urine culture results cannot be used to discriminate APN among children with fUTI.

\section{Blood C-reactive protein, erythrocyte sedimentation rate, and WBC are very important factors for discriminating APN among children with fUTI}

Blood C-reactive protein (CRP), erythrocyte sedimentation rate (ESR), and WBC have higher sensitivity and specificity for discriminating APN among children with fUTI than urine culture. ${ }^{3)}$ Blood sampling is less invasive and reveals its results more promptly than the urethral catheterization for urine culture. Therefore, the former would be a better choice for discriminating APN. CRP is positively correlated with the area of cortical defects on their DMSA results. Procalcitonin is more sensitive for detecting APN and renal scarring than CRP but is expensive; in practice, the combination of CRP, $\mathrm{ESR}$, and WBC is not worse than procalcitonin. ${ }^{10)}$ Plasma neutrophil gelatinase-associated lipocalin is not practical as a marker of APN in children.

The use of urine culture and antibiotic sensitivity tests is necessary to identify causative organisms. However, it is well known that most of the causative organisms (95\%) of UTI are gram-negative bacteria. The empirical antibiotic treatment of UTI is typically successful in children with APN, particularly those less than 24 months of age at the first episode of UTI, even though extended-spectrum $\beta$-lactamase-positive bacteria have been revealed. If fever in a child suspected of APN does not subside within 48 hours after the start of antibiotic treatment, antibiotics would be added or exchanged before the urine culture results were revealed. Even though the urine culture sample would be contaminated, antibiotic-resistant organisms would be revealed in urine culture plates.

\section{Proteinuria and urine culture results have similar sensitivities for predicting UTI}

Proteinuria is detected in about 70\%-90\% of children with UTI. ${ }^{11,12)}$ However, its pathogenesis remains unknown. The authors assumed that the activation of Toll-like receptor in the uroepithelium would cause proteinuria in UTI like in immunoglobulin A nephropathy or diabetic nephropathy. ${ }^{13,14)}$ Proteinuria in children with UTI is significantly correlated with positive urine culture results. ${ }^{3)}$ Children with aseptic pyuria and asymptomatic bacteriuria do not have proteinuria.

\section{Pyelitis does not lead to renal scarring}

There are three types of fUTI: pyelitis, APN, and lower UTI with 
another fever focus. Pyelitis is defined as an ascending infection in the renal pelvis without involvement of the renal parenchyma. There is no major difference in the clinical and laboratory findings of pyelitis and APN. The former shows a normal DMSA scan result along with lower CRP, ESR, and WBC than the latter. ${ }^{12)}$ Therefore, pyelitis will not progress to acquired renal scarring if it is detected as early as possible and its treatment is initiated with oral antibiotics. In the same texture, children with recurrent pyelitis do not urgently need to undergo an evaluation of urinary tract anomalies because of the absence of acquired renal scarring. Urine culture results are not helpful for discriminating APN among these three types of fUTI.

\section{DMSA scan or renal computed tomography can confirm APN or renal scarring}

The acute DMSA scan is most commonly performed to confirm APN among the 3 types of fUTI. However, its use is not appropriate whenever APN is suspected due to radiation exposure and sedative use. Renal computed tomography is more sensitive for detecting cortical defects than a DMSA renal scan, but its radiation exposure is about 10 times higher.

The positive findings observed in acute DMSA scan lead us to perform a follow-up DMSA scan to check for the development of acquired renal scarring. Thus, the exposed radiation dose would be doubled. Considering the long-term prognosis of APN, the presence of acquired renal scarring is a more important factor than discriminating APN at the diagnosis of an acute infection, and the presence of congenital hypoplastic kidney is a more important factor than the presence of acquired renal scarring or discriminating APN. Thus, it is necessary to perform a DMSA scan in children with recurrent fUTI.

\section{VUR operation is not immediately necessary in children without renal scarring}

Only 20\%-25\% of high-grade VUR cases spontaneously disappear within 10 years after detection. Among the congenital anomalies of the kidney and urinary tract, high-grade VUR is the most common cause of recurrent APN. Therefore, high-grade VUR threatening diminished renal function should be treated as soon as possible. Otherwise, high-grade VUR is benign except in some children suffering from recurrent fUTI or bladder symptoms. Therefore, if APN does not recur, the early detection of VUR is unnecessary. However, high-grade VUR associated with congenital hypoplastic kidney or congenital renal scarring should be detected as early as possible.

In our previous study, only 16 of 618 children (2.6\%) admitted with a diagnosis of the first episode of APN between 2000 and 2009 underwent a VUR operation after UTI imaging studies. ${ }^{15)}$

\section{UTI imaging study guideline}

The UTI imaging guidelines of the American Academy of Pediatrics (AAP) used worldwide are summarized as follows: First, if a febrile ill-looking infant without apparent fever focus visits a clinic, clinicians should perform urethral catheterization to obtain urine samples for culture and urinalysis. (Urethral catheterization is an important procedure for diagnosing UTI.) Second, febrile infants with UTI should undergo renal and bladder ultrasonography (RBUS). Third, voiding cystourethrography (VCUG) should not be performed routinely after the first febrile UTI. VCUG is indicated if the RBUS reveals hydronephrosis, scarring, or other findings that would suggest high-grade VUR or obstructive uropathy as well as in other atypical or complex clinical circumstances. Further evaluations should be conducted if fUTI recurs. ${ }^{16)}$

The AAP also stated the following limitations in its report. First, the development of techniques that would permit an alternative to invasive sampling and culture would be valuable for general use. Second, incubation time, which is inherent in the culture process, results in delayed or presumptive treatment. Third, the role of VUR is incompletely understood. It is not supported that antimicrobial prophylaxis is beneficial for VUR patients to prevent UTI recurrence. Fourth, this guideline is limited to the initial management of the first UTI in febrile infants 2-24 months of age. No strategy for children with recurrent UTI is currently used. Furthermore, AAP guidelines did not contain a strategy for children with congenital or acquired renal scarring that to lead to CKD.

It is important that we focus on APN, as opposed to other fUTI, along with the detection of congenital or acquired renal scarring. The presence of high-grade VUR requires consideration because

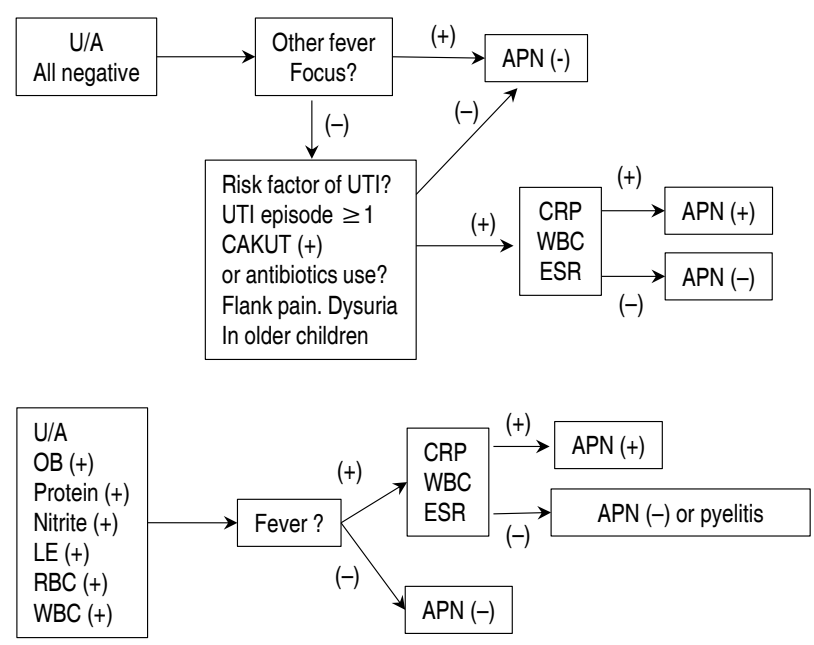

Fig. 1. Diagram of acute pyelonephritis (APN) discrimination at the first visit. U/A, urinalysis; UTI, urinary tract infection; CAKUT, congenital anomalies of kidney and urinary tract; CRP, C-reactive protein; WBC, white blood cell; ESR, erythrocyte sedimentation rate; OB, occult blood; $\mathrm{LE}$, leukocyte esterase; RBC, red blood cell. 
urgent surgical treatment is not indicated in cases with normal DMSA results regardless of the previous number of recurrent UTI. ${ }^{13)}$ It is important that a DMSA scan be performed regardless of the urine culture result in children with a second episode of APN, pyelitis, recurrent atypical UTI (more than three times per year), or small kidney on ultrasonograph. Thus, children with normal DMSA scan results do not urgently require VCUG.

In our study, 60\%-70\% of children with a second episode of APN showed a normal DMSA scan result performed 3 months after its episode when they had the first episode of APN history at less than 24 months of age.

\section{Older children at the first APN episode diagnosis}

Older children with APN generally suffer from more severe symptoms (prolonged fever, abdominal pain, or flank pain) than young children. If they have risk factors like hydronephrosis on renal ultrasound or severe disease progression, a DMSA scan is recommended 6 months later to evaluate the presence of renal scarring.

\section{New challenging method to discriminate APN among children with fUTI}

The results of our method for discriminating APN using CRP, ESR, and WBC as opposed to urine culture result are outlined in Figs. 1 and 2. A hypothetical diagram of the pathogenesis of APN is shown in Fig. 3. ${ }^{8)}$

The intrarenal local renin-angiotensin-aldosterone system may be activated in children with APN. The antinatriuretic effect of increased intrarenal angiotensin II causes increased tubular reabsorption of sodium and increased tubular secretion of potassium. Thus, the urine sodium level and urine sodium to potassium

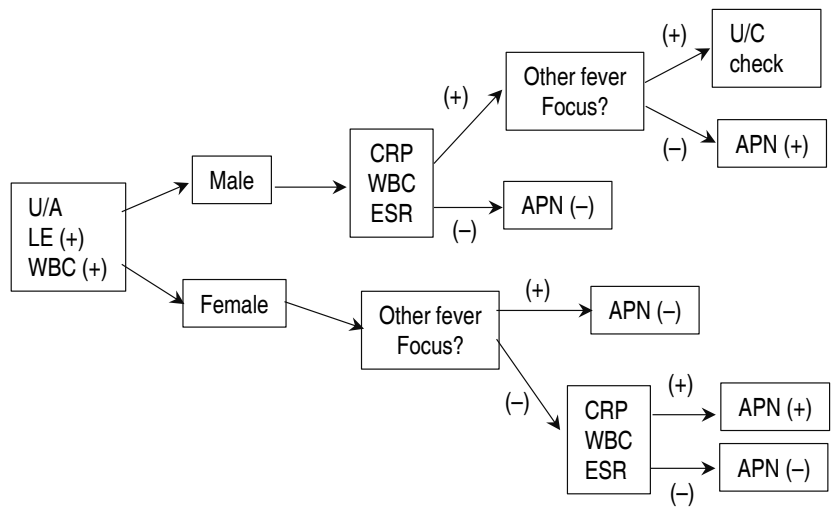

Fig. 2. Diagram of acute pyelonephritis (APN) discrimination in children with only pyuria on urinalysis. U/A, urinalysis; LE, leukocyte esterase; WBC, white blood cell; CRP, C-reactive protein; ESR, erythrocyte sedimentation rate; $\mathrm{U} / \mathrm{C}$, urine culture. ratio decrease.

A new challenging method to discriminate APN among children with fUTI is shown in Fig. 4.

\section{Conclusion}

The usefulness of urine culture results is very limited for patients with complicated UTI or antibiotic unresponsiveness. Furthermore, the usefulness of the diagnostic purpose is low since urine culture results are revealed a few days after the measurements are initiated and do not suggest the location of the UTI. Other types of information, such as age, sex, past medical history, urinalysis, blood CRP level, ESR, and WBC are as important as urine culture and considered important in the diagnosis of APN in children.

The long-term prognosis of APN in young children, especially infants, depends on the number of recurrent APN, not recurrent fUTI. If APN does not recur even once, no medical or surgical treatment or imaging studies are needed, even if a patient has congenital hypoplastic kidney or high-grade VUR, because the further progression of acquired renal scarring will not occur. There are $2 \mathrm{im}$ portant factors that pediatricians should not overlook: whether APN is recurrent ( $90 \%$ of children with the second episode of APN recurred within 1 year after the first episode of APN), and a DMSA scan should be performed in children with recurrent APN to prevent missing an observation of congenital hypoplastic kidney.

New methods that can diagnose APN in children promptly, practically, and comfortably should be identified in the future as alternatives to urinary catheterization for urine culture sampling.

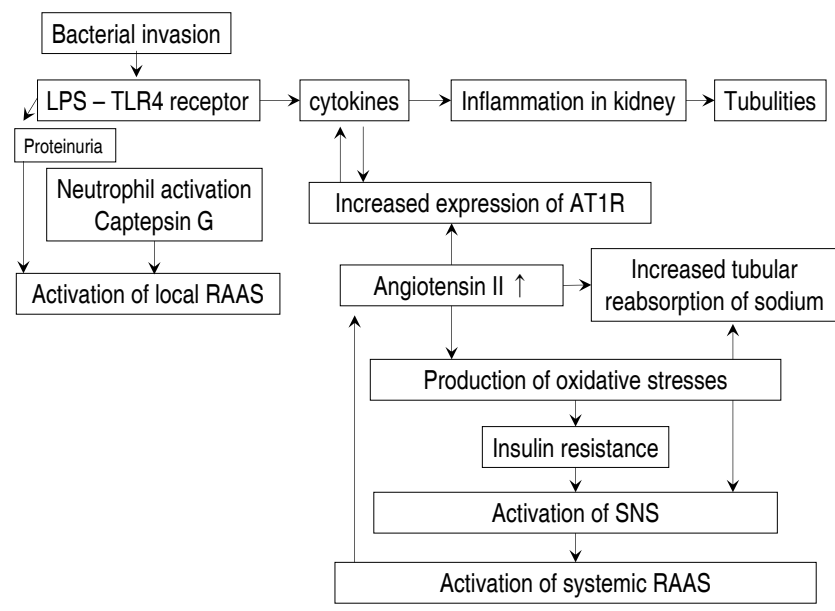

Fig. 3. Hypothetical diagram of the pathogenesis of acute pyelonephritis. LPS, lipopolysaccharide; TLR 4, toll-like receptor 4; RAAS, renin-angiotensin-aldosterone system; AT1R, angiotensin II receptor type 1; SNS, sympathetic nervous system. 




Fig. 4. Diagram of discriminating APN from fUTI using new method. Positive results of CRP, WBC, and ESR indicate upper urinary tract infection including acute pyelitis or pyelonephritis (APN). The higher those values are, the more probable APN is. A urine protein to creatinine ratio greater than 0.5 indicates the possibility of the presence of significant bacteriuria. Decreased urine sodium level $(<40$ $\mathrm{mEq} / \mathrm{L})$ indicates the possibility of APN. A decreased urine sodium to potassium ratio $(<1.15)$ indicates the possibility of APN but has a lower correlation with APN than the other factors. U/A, urinalysis; LE, leukocyte esterase; WBC, white blood cell count; CRP, C-reactive protein; ESR, erythrocyte sedimentation rate; PU, urine protein to creatine ratio; uNa, urine sodium $(\mathrm{mg} / \mathrm{dL}) ; \mathrm{uNa} / \mathrm{K}$, urine sodium to potassium ratio; AP, acute pyelitis; f/u, follow-up.

\section{Conflicts of interest}

No potential conflict of interest relevant to this article was reported.

\section{References}

1. Lee KY. New insights for febrile urinary tract infecton (acute pyelon ephritis) in children. Child Kidney Dis 2016;20:37-44.

2. Nammalwar BR, Vijayakumar M, Sankar J, Ramnath B, Prahlad N. Evaluation of the use of DMSA in culture positive UTI and culture negative acute pyelonephritis. Indian Pediatr 2005;42:691-6.

3. Lee JH. Discrimination of culture negative pyelonephritis in children with suspected febrile urinary tract infection and negative urine culture results. J Microbiol Immunol Infect 2019;52:598-603.

4. Leman P. Validity of urinalysis and microscopy for detecting urinary tract infection in the emergency department. Eur J Emerg Med 2002;9:141-7.

5. Ipe DS, Sundac L, Benjamin WH Jr, Moore KH, Ulett GC. Asymptomatic bacteriuria: prevalence rates of causal microorganisms, etiology of infection in different patient populations, and recent advances in molecular detection. FEMS Microbiol Lett 2013;346:1-10.

6. Pokrajac D, Sefic-Pasic I, Begic A. Vesicoureteral reflux and renal scarring in infants after the first febrile urinary tract infection. Med Arch 2018;72:272-5.

7. Lugg-Widger FV, Angel L, Cannings-John R, Jones H, Lau M, Butler C, et al. Long-term outcomes of urinary tract infection (UTI) in childhood (LUCI): protocol for an electronic record-linked cohort study. BMJ Open 2019;9:e024210.
8. Warady BA, Chadha V. Chronic kidney disease in children: the global perspective. Pediatr Nephrol 2007;22:1999-2009.

9. Lee JH, Jang SJ, Rhie S. Antinatriuretic phenomena seen in children with acute pyelonephritis may be related to the activation of intrarenal RAAS. Medicine (Baltimore) 2018;97:e12152.

10. Shaikh N, Borrell JL, Evron J, Leeflang MM. Procalcitonin, C-reactive protein, and erythrocyte sedimentation rate for the diagnosis of acute pyelonephritis in children. Cochrane Database Syst Rev 2015 Jan 20;1:CD009185. https://doi.org/10.1002/14651858.CD009185.pub2.

11. Eidelman Y, Raveh D, Yinnon AM, Ballin J, Rudensky B, Gottehrer NP. Reagent strip diagnosis of UTI in a high-risk population. Am J Emerg Med 2002;20:112-3.

12. Jung SJ, Lee JH. Prediction of cortical defect using c-reactive protein and urine sodium to potassium ratio in infants with febrile urinary tract infection. Yonsei Med J 2016;57:103-10.

13. Verzola D, Cappuccino L, D'Amato E, Villaggio B, Gianiorio F, Mij M, et al. Enhanced glomerular Toll-like receptor 4 expression and signaling in patients with type 2 diabetic nephropathy and microalbuminuria. Kidney Int 2014;86:1229-43.

14. Coppo R, Camilla R, Amore A, Peruzzi L, Daprà V, Loiacono E, et al. Toll-like receptor 4 expression is increased in circulating mononuclear cells of patients with immunoglobulin A nephropathy. Clin Exp Immunol 2010;159:73-81.

15. Lee JH, Kim MK, Park SE. Is a routine voiding cystourethrogram necessary in children after the first febrile urinary tract infection? Acta Paediatr 2012;101:e105-9.

16. Subcommittee on Urinary Tract Infection, Steering Committee on Quality Improvement and Management, Roberts KB. Urinary tract infection: clinical practice guideline for the diagnosis and management of the initial UTI in febrile infants and children 2 to 24 months. Pediatrics 2011;128:595-610. 